Präv Gesundheitsf 2021 · 16:215-224

https://doi.org/10.1007/s11553-020-00809-6

Eingegangen: 27. April 2020

Angenommen: 1. August 2020

Online publiziert: 26. August 2020

(c) Der/die Autor(en) 2020

\section{Einleitung}

Bei Lehrkräften sind die hohe psychische Belastung sowie die daraus resultierende schlechte gesundheitliche Situation schon seit langem bekannt $[2,5,16$, 28, 30].

$\mathrm{Zu}$ den Belastungsfaktoren zählen konkrete Arbeitsbedingungen wie Zeitdruck, große Schulklassen, die Arbeit mit undisziplinierten, teils aggressiven oder unmotivierten Schülern ${ }^{1}$, aber auch neue Bedingungen wie die Nutzung von Informationstechnologien infolge der Digitalisierung. Hinzu kommen psychoemotionale Belastungen wie erlebte verbale Gewalt, ein zunehmend multikulturelles Sozialleben, Ausfüllen unterschiedlicher Rollen (Pädagoge, Erzieher, Sozialarbeiter, Vermittler) sowie die hohe Verantwortung gegenüber den Schülern, die Arbeit im Kollegium als auch mit Eltern [30]. Nicht zuletzt belastet der erhebliche Lärm in den Klassenräumen, v.a. im Sportunterricht, aber auch in den Pausen und auf dem Schulhof (Pausenaufsicht), was zu Hörschäden, vermehrter Stimmbelastung und mentaler Ermüdung führen kann [20, 31].

Aus einer dauerhaft erhöhten Belastung können Fehlbeanspruchungen in Form von verschiedenen Erkrankungen resultieren. Seibt et al. [32] sowie auch Borelli et al. [5] verbinden die hohen Anforderungen im Lehrerberuf mit einer vermehrten Rate an Depressionen. Die Depressionsrate fällt umso höher aus, je geringer die soziale Unterstützung aus

\footnotetext{
1 In dieser Arbeit wird aus Gründen der besseren Lesbarkeit die männliche Sprachform verwendet, es sind aber, wenn nicht explizit angegeben, immerbeide Geschlechtergemeint.
} Deutschland

\title{
Psychische Gesundheit bei Referendaren
}

dem Umfeld ist. Die Länge der Arbeitszeit scheint dagegen den Gesundheitszustand nicht zu beeinflussen. Seibt et al. [33] konstatierten bei einem Fünftel sowohl teil- als auch vollzeitbeschäftigter Lehrkräfte eine gleichermaßen eingeschränkte psychische Gesundheit. Dagegen litten Hauptschullehrer eher an einer verminderten psychischen Gesundheit als Gymnasiallehrer [2].

Auch Shimizu et al. [34] stellten den Zusammenhang zwischen chronischer Ermüdung und belastenden Arbeitsbedingungen fest. Chronische Müdigkeit und mangelnde Erholung hatten in einer Studie an finnischen Lehrern neben einem Ungleichgewicht zwischen Arbeitsanforderungen und Belohnung die Entwicklung eines Burnout-Syndroms mit begünstigt [14].

In einer großen, länderübergreifenden Studie an über 90.000 Probanden untersuchten Dragano et al. [12] den Einfluss von (zu) hohen Arbeitsanforderungen bei gleichzeitig (zu) geringer Belohnung auf die Entwicklung von HerzKreislauf-Erkrankungen(HKE).Zu hohe Anforderungen erhöhen das HKE-Risiko um $16 \%$, bei gleichzeitig zu gering empfundener Belohnung steigt das Risiko um $41 \%$. Darüber hinaus besteht ein Zusammenhang zwischen vermehrtem psychosozialen Stress und einer Depression. Insbesondere erhöhten hohe Anforderungen und geringe Entscheidungsfreiheit das Depressionsrisiko [4].

Zwar ist der Anteil der Frühberentungen seit 2001 aufgrund geänderter gesetzlicher Rahmenbedingungen stetig rückläufig, jedoch ist der Anteil frühpensionierter Lehrer aufgrund von Dienstunfähigkeit mit 12-13\% immer noch zu hoch. Nur etwa ein Drittel der 2017 pen- sionierten Lehrkräfte schied mit Erreichen der gesetzlichen Regelaltersgrenze aus dem Schuldienst aus [9, 11].

Vedovato und Monteiro [36] stellten einen signifikanten Zusammenhang zwischen der subjektiv eingeschätzten Arbeitsfähigkeit und dem Alter sowie den Berufsjahren als Lehrer fest. Zumeist waren es die älteren Lehrkräfte, die über eine verminderte Arbeitsfähigkeit verfügten. Jedoch fanden die Autoren in der untersuchten Stichprobe auch unter den jüngeren Probanden (unter 30 Jahre) Lehrerinnen mit einer geringen bzw. moderaten Arbeitsfähigkeit.

Nicht nur aus diesem Grund ist es wichtig, junge Menschen wie Studierende oder Auszubildende in den Mittelpunkt der Forschung zu stellen. Bailer et al. [1] stellten in einer Befragung unter Studierenden einer Universität bei knapp $23 \%$ der Teilnehmenden bereits psychische Erkrankungen wie somatoforme Störungen oder auch depressive Syndrome fest. Auch unter Medizinstudierenden wurden psychische Störungen wie emotionale Erschöpfung und eine höhere Burnout-Rate aufgrund zu hoher Belastung beobachtet [3]. Brandl-Bredenbeck et al. [6] untersuchten die Gesundheit von Lehramtsstudierenden und stellten eine im Vergleich zur Normstichprobe beeinträchtigte psychische Gesundheit fest, insbesondere bei weiblichen Studierenden in Prüfungssituationen. Über $21 \%$ der Lehramtsstudierenden der Universität Frankfurt weisen arbeitsbedingte Verhaltens- und Erlebensmuster auf, die auf ein Burnout-Risiko hindeuten [26]. Im Referendariat kommen dann weitere Belastungsfaktoren wie das Erlernen der Kompetenzen in den Bereichen Unterrichten, Erziehen oder Beurteilen hinzu 


\begin{tabular}{|c|c|c|}
\hline Variable & Gesamt $(n=131), M W \pm S D$ & $p_{\text {Mann-Whitney }}$ \\
\hline Alter (Jahre) & $28,1 \pm 3,70$ & 0,003 \\
\hline Weiblich $(n=102)$ & $27,7 \pm 3,79$ & \\
\hline Männlich ( $n=29)$ & $29,4 \pm 3,03$ & \\
\hline \multicolumn{2}{|c|}{ Psychische Gesundheit (GHQ-12-Punktwert) } & 0,309 \\
\hline Weiblich & $12,5 \pm 6,59$ & \\
\hline Männlich & $13,2 \pm 5,22$ & \\
\hline \multicolumn{2}{|c|}{ Overcommitment (Punktwert) } & 0,565 \\
\hline Weiblich & $15,5 \pm 3,74$ & \\
\hline Männlich & $14,9 \pm 4,29$ & \\
\hline \multicolumn{2}{|c|}{ Burnout-Risiko nach Kalimo (Punktwert) } & 0,461 \\
\hline Weiblich & $1,32 \pm 0,92$ & \\
\hline Männlich & $1,33 \pm 0,68$ & \\
\hline
\end{tabular}

[13]. Christ et al. [8] beschrieben diese Phase der Lehrerausbildung als stark belastend.

Nach Abschluss des Lehramtsstudiums (1. Staatsexamen) beginnt die Phase des Referendariats, die ehemals Studierenden werden nun als Referendare bzw. Lehramtsanwärter bezeichnet. An einem Tag in der Woche verbringen die Referendare im „Staatlichen Seminar für Lehrämter", wo sie noch einmal Theorie (aber mit mehr Praxisbezug als im Studium) vermittelt bekommen. An 4 von 5 Tagen in der Woche werden sie in den Schulen eingesetzt. In Sachsen-Anhalt ist das Referendariat in drei Phasen untergliedert: In der Einstiegsphase am Anfang (mit einer Dauer von in der Regel 4 Monaten) hospitieren die Referendare oftmals, diese Phase dient der Einführung in die schulpraktische Tätigkeit. In der Qualifizierungsphase (in der Regel 8 Monate) erfolgt die weitere Profilierung und Qualifizierung im jeweiligen Lehramt. Die Referendare unterrichten selbst und übernehmen Aufsichten. In der abschließenden Prüfungsphase wird die Laufbahnprüfung abgelegt (2. Staatsexamen).

Ziel der Studie war es zu klären, ob und inwieweit die Gesundheit von Lehramtsanwärtern im zweiten Ausbildungsabschnitt in Abhängigkeit von der Referendariatsphase sowie von individuellen arbeitsbezogenen Verhaltens- und Erlebensmustern bereits beeinträchtigt ist, um den Bedarf an psychotherapeutischen Beratungen, Stressbewäl- tigungskursen o. ä. Interventionen an den Hochschulen bzw. an den Schulen einzuschätzen.

\section{Probanden und Methodik}

Die Fragebogenerhebung fand im Jahr 2016 in Magdeburg statt im Anschluss an eine größere Befragung bei Lehrkräften im Rahmen eines von der Deutschen Gesetzlichen Unfallversicherung (DGUV) geförderten Projekts „Im Lehrerberuf gesund und munter bis zur Rente - Wege der Prävention und Personalentwicklung“. An der Studie nahmen 131 Lehramtsanwärter im Alter von 28,1 \pm 3,7 Jahren aus Magdeburg und Umgebung freiwillig teil, die sich nach Bestehen des 1. Staatsexamens in verschiedenen Phasen des Referendariats befanden. Bei den Befragten waren alle Schulformen vertreten: Lehramt Gymnasium, Grund-, Förder- und Sekundarschule sowie Berufsbildende Schulen. Die Fragebogenerhebung fand während eines Pflichtseminars statt. Alle ausgeteilten Fragebögen wurden direkt im Anschluss eingesammelt. Von allen Referendaren lag ein vollständiger Datensatz vor.

Zur Erfassung des arbeitsbezogenen Verhaltens- und Erlebensmusters (AVEM) wurde der gleichnamige Fragebogen von Schaarschmidt und Fischer [29] verwendet. Mithilfe von 66 Items können Rückschlüsse auf einen gesundheitsförderlichen bzw. -gefährdenden Umgang mit Anforderungen im Beruf sowie persönlichen Ressourcen gezogen werden. Anhand von 11 Dimensionen können vier Muster unterschieden werden: die gesundheitsförderlichen Muster G (Gesundheit) und S (Schonung) sowie die gesundheitsgefährdenden Muster A (Anstrengung; Anlehnung an Typ-A-Verhalten) und B (BurnoutGefährdung). Eine Zuordnung zu den jeweiligen Mustern wurde nach Schaarschmidt und Fischer [29] bei einer mindestens $60 \%$ igen Wahrscheinlichkeit vorgenommen.

Die psychische Gesundheit wurde mit der Kurzversion des „General Health Questionnaire 12“(GHQ-12; [15]) erhoben. In 12 Items wird nach kurzfristigen Beeinträchtigungen des Gesundheitszustands in den letzten Wochen gefragt. Die Auswertung erfolgte in der dichotomen Variante (0-0-1-1-Kodierung) nach Goldberg. Ein Cut-off-Wert von 5 wurde nach Linden et al. [22] verwendet und die Studienteilnehmer entsprechend in die Gruppen mit „normaler psychischer Gesundheit“ und „beeinträchtigter psychischer Gesundheit“ eingeteilt.

Die Ausprägung der Burnout-Symptomatik wurde mit dem „Maslach Burnout Inventory“ (MBI-GS; [23]) erhoben und das Burnout-Risiko nach Kalimo et al. [17] klassifiziert. Mithilfe von 16 Fragen wird in den drei Kategorien „emotionale Erschöpfung“, „Zynismus“ und „Leistungsfähigkeit“ die Ausprägung der Burnout-Symptomatik ermittelt. Für eine Einschätzung des individuellen Burnout-Risikos erfolgte anschließend die Berechnung des BurnoutRisikos nach Kalimo et al. [17]. Bei einem Punktwert $<1,49$ besteht kein BurnoutRisiko. Zwischen 1,5-3,49 Punkte sind einige Burnout-Symptome mehrmals im Monat vorhanden. Liegt der errechnete Wert $>3,5$ Punkten, besteht ein BurnoutRisiko.

Zur Erfassung der Verausgabungsbereitschaft wurde der Fragebogen zur „effort-reward imbalance“ (ERI) in der Kurzform mit 10 Fragen inklusive der Subskala „overcommitment“ (OC) mit weiteren 6 Fragen nach Siegrist [35] genutzt. Zur Berechnung der ERI wurde der Summenwert von der Skala „effort" durch den Summenwert der Skala „reward“ dividiert und wegen der unterschiedlichen Anzahl an Items mit 
einem Korrekturfaktor multipliziert. Bei einem Wert $>$ „1“ ist ein Ungleichgewicht zwischen Anforderung und Belohnung vorhanden. Bei der Skala für das OC wird ab einem Summenwert $>18$ eine erhöhte Anstrengungsbereitschaft konstatiert.

Die statistische Auswertung der Rohdaten erfolgte mit dem Statistik- und Analyse-Programm SPSS, Version 26 (IBM, Armonk, New York, USA). Zunächst wurden deskriptive Analysen durchgeführt. Mittels exaktem FisherTest wurden Unterschiede bei kategorialen Daten berechnet. Für die Analysen von Unterschieden zwischen den Referendaren in den verschiedenen Referendariatsphasen wurde der MannWhitney-Test bei intervallskalierten, nicht normalverteilten Variablen verwendet. Bei normalverteilten Variablen wurde eine einfaktorielle Varianzanalyse mit Bonferroni-Test (post-hoc) genutzt. Ein Signifikanzniveau von $p<0,05$ liegt allen Testverfahren zugrunde.

\section{Ergebnisse}

Unter den 131 Referendaren, die sich an der Fragebogenerhebung beteiligten, befanden sich 29 Männer im Alter von 29,4 $\pm 3,0$ Jahren und 102 Frauen (27,8 $\pm 3,8$ Jahre). Die Männer waren signifikant 1,6 Jahre älter als die Frauen $(p<0,05)$. Der Vergleich zwischen Männern und Frauen hinsichtlich des Burnout-Risikos, der psychischen Gesundheit sowie des OC ergab keine signifikanten Unterschiede (• Tab. 1), sodass Männer und Frauen in dieser Studie als eine Gesamtstichprobe behandelt und nur die einzelnen Phasen des Referendariats beschrieben werden.

In der Einstiegsphase des Referendariats befanden sich 21 (29\%) Männer und 51 (71\%) Frauen, in der Qualifizierungsphase 8 (16\%) Männer und 43 (84\%) Frauen und in der Prüfungsphase waren 8 (100\%) Frauen. Innerhalb der einzelnen Phasen des Referendariats sind Männer und Frauen gleich verteilt $(p=0,062)$. In Bezug auf das Alter unterscheiden sich die Männer in den jeweiligen Referendariatsphasen nicht von den Frauen.

Die Tab. 2 gibt einen Überblick über die Gesamtergebnisse der Fragebogenerhebung in den einzelnen Phasen des Re-

Präv Gesundheitsf 2021 · 16:215-224 https://doi.org/10.1007/s11553-020-00809-6

(c) Der/die Autor(en) 2020

S. Darius · K. Bunzel · E. Ehms-Ciechanowicz • I. Böckelmann

Psychische Gesundheit bei Referendaren

\section{Zusammenfassung}

Hintergrund. Lehrkräfte sind in ihrem Beruf verschiedenen Belastungen ausgesetzt, aufgrund derer es zu Fehlbeanspruchungen kommen kann. Weniger bekannt ist dagegen die gesundheitliche Situation bei Referendaren.

Ziel der Studie. Es war zu klären, ob und inwieweit die psychische Gesundheit in Abhängigkeit der Referendariatsphase bei Referendaren beeinträchtigt ist, um den Bedarf an Präventionsmaßnahmen einschätzen zu können.

Methodik. Für die Studie wurden 131 Referendare im Alter von 28,1 $\pm 3,7$ Jahren aus Magdeburg und Umgebung befragt. 72 von innen befanden sich in der Einstiegsphase des Referendariats, 51 in der Qualifizierungsphase und 8 in der Prüfungsphase. Die psychische Gesundheit wurde mit der Kurzversion des "General Health Questionnaire 12" (GHQ12), die Anstrengungsbereitschaft mit einem Teil des Effort-reward-imbalance(ERI)Fragebogens und das Burnout-Risiko mit dem Maslach-Burnout-Inventar (MBI) erhoben. Zusätzlich wurden arbeitsbezogene
Verhaltens- und Erlebensmuster (AVEM) erfasst.

Ergebnisse. Bei 41 (31,3\%) Referendaren war die psychische Gesundheit bereits beeinträchtigt, sie wiesen auch einige BurnoutSymptome auf. In der Qualifizierungsphase war die emotionale Erschöpfung stärker als in der Einstiegsphase. Mehrheitlich waren gesundheitsförderliche Muster G und S vertreten, bei etwa einem Drittel der Referendare überwogen die gesundheitsgefährdenden Verhaltensmuster A und B.

Schlussfolgerung. Da sich die psychische Gesundheit bereits im Referendariat bei etwa einem Drittel der Referendare beeinträchtigt gezeigt hat, müssen präventive Maßnahmen als Pflichtveranstaltung in das Studium integriert werden, um die Gesundheit der Referendare zu erhalten.

\section{Schlüsselwörter}

Lehramtsanwärter · General health questionnaire - Burnout-Risiko · Arbeitsbezogene Verhaltens- und Erlebensmuster . Referendariat

\section{Mental health among student teachers}

\section{Abstract}

Background. In their profession, teachers are exposed to various stresses, which may lead to impaired mental health. The health situation among student teachers is less well known.

Objectives. The aim of the study was to clarify whether and to what extent mental health is impaired depending on the referee phase in student teachers, in order to be able to assess the need for prevention measures.

Methods. For the study, 131 student teachers aged 28.1 \pm 3.7 years from Magdeburg and the surrounding area were interviewed: 72 students were in the entry phase of the teacher traineeship, 51 in the qualification phase and 8 in the examination phase. Mental health was assessed with the short version of the General Health Questionnaire (GHQ-12), overcommitment with the part of the Effort Reward Imbalance Questionnaire (ERI) and the risk of burnout with the Maslach Burnout
Inventory (MBI). Work-related behaviour and experience patterns (AVEM) were recorded. Results. Mental health was already impaired in 41 (31.3\%) student teachers, and they also had burnout symptoms. In the qualification phase, emotional exhaustion is higher than in the entry phase. The majority of students had health-promoting patterns $\mathrm{G}$ and $\mathrm{S}$, and approximately a third of the students had predominantly health-threatening behaviour patterns $A$ and $B$.

Conclusion. Mental health is already impaired in the teacher training in approximately a third of the students. Therefore, preventive measures must be integrated into the course as a compulsory event in order to maintain the health of the students.

\section{Keywords}

Student teachers - General health questionnaire $\cdot$ Risk of burnout - Work-related behavior and experience $\cdot$ Teacher traineeship 


\section{Originalarbeit}

Tab. 2 Übersicht über die Ergebnisse der Fragebogenerhebung in den einzelnen Phasen des Referendariats unter Einbeziehung des Alters als Kovariable

\begin{tabular}{|c|c|c|c|c|c|c|c|}
\hline Parameter & $\begin{array}{l}\text { Einstiegs- } \\
\text { phase (I), } \\
\text { MW } \pm \text { SD }\end{array}$ & $\begin{array}{l}\text { Qualifizierungs- } \\
\text { phase (II), MW } \pm \text { SD }\end{array}$ & $\begin{array}{l}\text { Prüfungs- } \\
\text { phase (III), } \\
\text { MW } \pm \text { SD }\end{array}$ & $\begin{array}{l}\text { Gesamtstich- } \\
\text { probe, MW } \pm S D\end{array}$ & $p_{\text {ANOVA }}$ & $p_{\text {Bonferroni }}$ & $\begin{array}{l}\text { Partielles Eta- } \\
\text { Quadrat (Alter) }\end{array}$ \\
\hline Psychische Gesundheit & $11,9 \pm 6,39$ & $13,3 \pm 5,78$ & $15,3 \pm 8,34$ & $12,7 \pm 6,23$ & 0,332 & - & 0,005 \\
\hline Overcommitment & $14,5 \pm 3,60$ & $16,6 \pm 4,01$ & $16,0 \pm 3,42$ & $15,4 \pm 3,87$ & 0,026 & $\begin{array}{l}0,008 \\
\text { (I vs. II) }\end{array}$ & $<0,001$ \\
\hline Emotionale Erschöpfung & $1,54 \pm 1,15$ & $1,87 \pm 1,25$ & $2,35 \pm 1,24$ & $1,72 \pm 1,21$ & 0,182 & - & 0,003 \\
\hline Zynismus & $0,80 \pm 0,99$ & $0,77 \pm 0,95$ & $1,3 \pm 1,32$ & $0,82 \pm 0,99$ & 0,575 & - & 0,002 \\
\hline Leistungsfähigkeit & $4,72 \pm 0,78$ & $4,74 \pm 0,76$ & $4,31 \pm 0,065$ & $4,70 \pm 0,77$ & 0,429 & - & 0,004 \\
\hline Burnout-Risikoscore & $1,24 \pm 0,86$ & $1,36 \pm 0,86$ & $1,83 \pm 0,89$ & $1,32 \pm 0,87$ & 0,311 & - & 0,001 \\
\hline
\end{tabular}

\begin{tabular}{|c|c|c|c|c|c|c|}
\hline Parameter & & $\begin{array}{l}\text { Einstiegsphase (I), } \\
n(\%)\end{array}$ & $\begin{array}{l}\text { Qualifizierungs- } \\
\text { phase (II), } n \text { (\%) }\end{array}$ & $\begin{array}{l}\text { Prüfungs- } \\
\text { phase (III), } n \text { (\%) }\end{array}$ & $\begin{array}{l}\text { Gesamtstich- } \\
\text { probe, } n(\%)\end{array}$ & $p_{\text {Fisher's exact Test }}$ \\
\hline \multirow{2}{*}{$\begin{array}{l}\text { Psychische } \\
\text { Gesundheit }\end{array}$} & Normal & $54(75,0)$ & $31(60,8)$ & $5(62,5)$ & $90(68,7)$ & \multirow[t]{2}{*}{0,227} \\
\hline & Beeinträchtigt & $18(25,0)$ & $20(39,2)$ & $3(37,5)$ & $41(31,3)$ & \\
\hline \multirow[t]{2}{*}{ Overcommitment } & Normal & $59(81,9)$ & $34(66,7)$ & $6(75,0)$ & $99(75,6)$ & \multirow[t]{2}{*}{0,133} \\
\hline & Erhöht & $13(18,1)$ & $17(33,3)$ & $2(25,0)$ & $32(24,4)$ & \\
\hline \multirow{3}{*}{$\begin{array}{l}\text { Emotionale } \\
\text { Erschöpfung }\end{array}$} & Gering & $50(69,5)$ & $34(66,7)$ & $5(62,5)$ & $89(67,9)$ & \multirow[t]{3}{*}{0,045} \\
\hline & Durchschnittlich & $16(22,2)$ & $6(11,8)$ & 0 & $22(16,8)$ & \\
\hline & Hoch & $6(8,3)$ & $11(21,6)$ & $3(37,5)$ & $20(15,3)$ & \\
\hline \multirow[t]{3}{*}{ Zynismus } & Gering & $54(75,0)$ & $38(74,5)$ & $5(62,5)$ & $97(74,0)$ & \multirow[t]{3}{*}{0,341} \\
\hline & Durchschnittlich & $13(18,1)$ & $6(11,8)$ & $1(12,5)$ & $20(15,3)$ & \\
\hline & Hoch & $5(6,9)$ & $7(13,7)$ & $2(25,0)$ & $14(10,7)$ & \\
\hline \multirow[t]{3}{*}{ Leistungsfähigkeit } & Gering & $13(18,1)$ & $12(23,5)$ & $2(25,0)$ & $27(20,6)$ & \multirow[t]{3}{*}{0,599} \\
\hline & Durchschnittlich & $24(33,3)$ & $14(27,5)$ & $4(50,0)$ & $42(32,1)$ & \\
\hline & Hoch & $35(48,6)$ & $25(49,0)$ & $2(25,0)$ & $62(47,3)$ & \\
\hline \multirow{3}{*}{$\begin{array}{l}\text { Klassifikation nach } \\
\text { Kalimo }\end{array}$} & Kein Burnout & $49(68,1)$ & $34(66,7)$ & $5(62,5)$ & $88(67,2)$ & \multirow[t]{3}{*}{0,980} \\
\hline & Einige Symptome & $22(30,6)$ & $16(31,4)$ & $3(37,5)$ & $41(31,3)$ & \\
\hline & Burnout-Risiko & $1(1,4)$ & $1(2,0)$ & 0 & $2(1,5)$ & \\
\hline
\end{tabular}

ferendariats. Dargestellt sind Mittelwerte mit der entsprechenden Standardabweichung. Hinsichtlich der psychischen Gesundheit und des Burnout-Risikos konnten keine Unterschiede in den einzelnen Phasen des Referendariats festgestellt werden. Lediglich die Referendare in der Qualifizierungsphase zeichneten sich durch eine leicht erhöhte Verausgabungsbereitschaft $(16,6 \pm 4,01$ Punkte) im Vergleich $\mathrm{zu}$ den Kollegen der Einstiegsphase $(14,5 \pm 3,6$ Punkte) aus $(p<0,01)$, wenngleich die Durchschnittswerte an sich mit 16,6 Punkten im Vergleich zu Normwerten nicht übermäßig erhöht waren.

Da sich die männlichen Referendare im Alter von den weiblichen Referendarinnen leicht unterschieden haben, wur- de das Alter bei den Tests als Kovariable mitberücksichtigt. Ein Einfluss des Alters ließ sich nicht feststellen.

Die psychische Gesundheit war bei 41 Referendaren der Gesamtstichprobe (31\%) bereits beeinträchtigt (• Tab. 3). In der Einstiegsphase waren $25 \%$, in der anschließenden Orientierungsphase knapp $40 \%$ und in der Prüfungsphase $37,5 \%$ der Referendare betroffen. Die Verausgabungsbereitschaft war bei 32 Referendaren erhöht. Insbesondere in der Qualifizierungsphase war ein Anstieg in den Punktwerten zu verzeichnen (- Tab. 2). Jedoch verschwindet der Unterschied nach Einteilung in die beiden Kategorien (normales und erhöhtes OC). Ein Zusammenhang zu den einzelnen Referendariatsphasen konnte somit nicht festgestellt werden. Ein ähnliches Ergebnis spiegelt sich auch bei der BurnoutSymptomatik wider. $31 \%$ der Teilnehmer klagten zumindest über einige BurnoutSymptome und bei $2 \%$ wurde ein Burnout-Risiko festgestellt. Insbesondere war die emotionale Erschöpfung der Referendare in der Qualifizierungsphase (21\% der Referendare) und in der Prüfungsphase $(37,5 \%)$ im Vergleich $\mathrm{zu}$ den Referendaren in der Einstiegsphase erhöht $(p<0,05)$.

Die Studienteilnehmer wiesen eine unterschiedliche Zugehörigkeit zu den AVEM auf. Insgesamt konnten 122 Referendare einem Muster zugeordnet werden. Mehrheitlich waren die gesundheitsförderlichen Muster $G$ und $S$ zu finden. Jedoch waren auch die Risiko- 


\begin{tabular}{|c|c|c|c|c|c|}
\hline Muster & $\begin{array}{l}\text { Einstiegsphase (I), } n \\
(\%)\end{array}$ & $\begin{array}{l}\text { Qualifizierungsphase (II), } n \\
\text { (\%) }\end{array}$ & $\begin{array}{l}\text { Prüfungsphase (III), } n \\
\text { (\%) }\end{array}$ & $\begin{array}{l}\text { Gesamtstichprobe, } n \\
\text { (\%) }\end{array}$ & $p_{\text {Fisher's exact Tes }}$ \\
\hline A & $7(9,7)$ & $9(17,6)$ & 0 & $16(12,2)$ & \multirow[t]{5}{*}{0,363} \\
\hline B & $17(23,6)$ & $12(23,5)$ & $1(12,5)$ & $30(22,9)$ & \\
\hline G & $18(25,0)$ & $13(25,5)$ & $3(37,5)$ & $34(26,0)$ & \\
\hline$S$ & $27(37,5)$ & $13(25,5)$ & $2(25,0)$ & $42(32,1)$ & \\
\hline $\begin{array}{l}\text { Ohne Zuord- } \\
\text { nung }\end{array}$ & $3(4,2)$ & $4(7,8)$ & $2(25,0)$ & $9(6,9)$ & \\
\hline
\end{tabular}

\begin{tabular}{|c|c|c|c|c|c|c|}
\hline Dimension & $\begin{array}{l}\text { Einstiegsphase (I), } \\
M W \pm S D\end{array}$ & $\begin{array}{l}\text { Qualifizierungs- } \\
\text { phase (II), MW } \pm \text { SD }\end{array}$ & $\begin{array}{l}\text { Prüfungsphase (III), } \\
M W \pm \text { SD }\end{array}$ & $\begin{array}{l}\text { Gesamtstich- } \\
\text { probe, } M W \pm S D\end{array}$ & $p_{\text {ANOVA }}$ & $p_{\text {Bonferroni }}$ \\
\hline $\begin{array}{l}\text { Subjektive Bedeutsamkeit der } \\
\text { Arbeit }\end{array}$ & $14,9 \pm 4,5$ & $14,9 \pm 4,3$ & $15,1 \pm 1,9$ & $14,9 \pm 4,3$ & 0,986 & - \\
\hline Beruflicher Ehrgeiz & $19,3 \pm 4,4$ & $19,4 \pm 4,3$ & $19,1 \pm 4,3$ & $19,4 \pm 4,3$ & 0,981 & - \\
\hline Verausgabungsbereitschaft & $17,0 \pm 4,5$ & $19,1 \pm 4,6$ & $18,9 \pm 5,5$ & $17,9 \pm 4,7$ & 0,049 & $\begin{array}{l}0,052 \\
\text { (I vs. II) }\end{array}$ \\
\hline Perfektionsstreben & $20,5 \pm 4,2$ & $19,8 \pm 5,4$ & $20,0 \pm 5,2$ & $20,2 \pm 4,7$ & 0,746 & - \\
\hline Distanzierungsfähigkeit & $17,5 \pm 5,2$ & $15,0 \pm 4,8$ & $16,7 \pm 3,5$ & $16,5 \pm 5,1$ & 0,022 & $\begin{array}{l}0,018 \\
\text { (I vs. II) }\end{array}$ \\
\hline Resignationstendenz & $16,0 \pm 4,6$ & $17,0 \pm 4,4$ & $15,4 \pm 3,5$ & $16,3 \pm 4,4$ & 0,404 & - \\
\hline $\begin{array}{l}\text { Offensive Problembewälti- } \\
\text { gung }\end{array}$ & $21,0 \pm 3,8$ & $21,5 \pm 3,5$ & $21,9 \pm 2,8$ & $21,2 \pm 3,6$ & 0,696 & - \\
\hline $\begin{array}{l}\text { Innere Ruhe und Ausgegli- } \\
\text { chenheit }\end{array}$ & $18,9 \pm 4,5$ & $19,5 \pm 4,3$ & $19,9 \pm 3,0$ & $19,2 \pm 4,3$ & 0,749 & - \\
\hline Erfolgserleben im Beruf & $22,8 \pm 3,2$ & $22,4 \pm 3,3$ & $23,4 \pm 3,7$ & $22,7 \pm 3,2$ & 0,676 & - \\
\hline Lebenszufriedenheit & $23,5 \pm 3,6$ & $23,3 \pm 3,4$ & $22,3 \pm 5,2$ & $23,3 \pm 3,6$ & 0,677 & - \\
\hline $\begin{array}{l}\text { Erleben sozialer Unterstüt- } \\
\text { zung }\end{array}$ & $25,6 \pm 3,1$ & $24,3 \pm 5,0$ & $24,6 \pm 5,3$ & $25,1 \pm 4,1$ & 0,212 & - \\
\hline
\end{tabular}

muster A (zu 13\%) und B (zu 24\%) vertreten. 9 Referendare konnten nicht eindeutig einem Muster zugeordnet werden (-Tab. 4). Ein Zusammenhang zur Referendariatsphase konnte nicht festgestellt werden $(p=0,297)$.

Innerhalb der einzelnen Subkategorien war lediglich eine sehr leicht erhöhte Verausgabungsbereitschaft der Referendare in der Qualifizierungsphase gegenüber denen in der Einstiegsphase $(19,1 \pm 4,6$ vs. $17,0 \pm 4,5$ Punkte, $p=0,052)$ sowie eine verminderte Distanzierungsfähigkeit $(15,0 \pm 4,8$ vs. $17,5 \pm 5,2$ Punkte, $p<0,05)$ festzustellen (• Tab. 5).

Bei differentieller Betrachtung innerhalb der einzelnen AVEM-Gruppen hinsichtlich normaler und beeinträchtigter psychischer Gesundheit ergaben sich signifikante Unterschiede $(p<0,001)$. Bei Referendaren mit Risikomustern A und B war die psychische Gesundheit in der Einstiegs- bzw. Qualifizierungsphase deutlich beeinträchtigter als bei Referendaren mit gesundheitsförderlichen Mustern G und S (•Tab.6). In der Prüfungsphase waren keine signifikanten Unterschiede festzustellen.

Referendare mit den Risikomustern A und B zeigten mehrheitlich auch eine erhöhte Verausgabungsbereitschaft (30,8 bzw. 46,2\% in der Einstiegsphase, $p<0,001)$ und der Qualifizierungsphase (29,4 bzw. 47,1\%, $p<0,01$; • Tab. 7). In der Prüfungsphase gab es keine signifikanten Unterschiede.

Ein ähnliches Bild zeigte sich hinsichtlich des Burnout-Risikos in Abhängigkeit von der Zugehörigkeit zu den AVEMMustern. Referendare mit dem Muster G wiesen in der Einstiegs- und Qualifizierungsphase kein Burnout-Risiko auf, nur in der Prüfungsphase traten bei ei- nem Probanden einige Burnout-Symptome auf. In der Einstiegsphase zeigten sich bei $71 \%$ der Referendare mit dem Risikomuster A einige Burnout-Symptome, in der Qualifizierungsphase dagegen nur bei $22 \%$. $65 \%$ der Referendare mit Risikomuster B wiesen in der Einstiegsphase Burnout-Symptome auf, in der Qualifizierungsphase waren es $58 \%(p<0,001$; - Tab. 8).

In der AVEM-Gruppe S traten bei $18 \%$ bereits einige Burnout-Symptome in der Einstiegsphase und später in der Qualifizierungsphase bei $38 \%$ auf. In der Prüfungsphase waren auch hier keine Unterschiede zu finden.

Ein signifikanter Zusammenhang wurde zwischen einigen arbeitsbedingten Verhaltens- und Erlebensmustern und der psychischen Gesundheit, dem OC sowie dem Burnout-Risiko gefunden (• Tab. 9). So besteht eine mittlere 
Tab. 6 Anzahl der Referendare mit entsprechender Musterzugehörigkeit mit normaler bzw. beeinträchtigter psychischer Gesundheit

\begin{tabular}{|c|c|c|c|c|c|c|c|}
\hline \multirow[t]{2}{*}{ Phase } & \multirow{2}{*}{$\begin{array}{l}\text { Psychische } \\
\text { Gesundheit }\end{array}$} & \multicolumn{5}{|c|}{ AVEM-Muster } & \multirow[t]{2}{*}{$p_{\text {Fisher's exact Test }}$} \\
\hline & & A, $n(\%)$ & $\mathrm{B}, \boldsymbol{n}(\%)$ & G, $n(\%)$ & $S, n(\%)$ & Keine Zuordnung, $\boldsymbol{n}$ (\%) & \\
\hline \multirow[t]{2}{*}{ Einstiegsphase } & Normal & $3(5,6)$ & $7(13,0)$ & $17(31,5)$ & $25(43,6)$ & $2(3,7)$ & \multirow[t]{2}{*}{$<0,001$} \\
\hline & Beeinträchtigt & $4(22,2)$ & $10(55,6)$ & $1(5,6)$ & $2(11,1)$ & $1(5,6)$ & \\
\hline \multirow{2}{*}{$\begin{array}{l}\text { Qualifizie- } \\
\text { rungsphase }\end{array}$} & Normal & $4(12,9)$ & $1(3,2)$ & $13(41,9)$ & $10(32,3)$ & $3(9,7)$ & \multirow[t]{2}{*}{$<0,001$} \\
\hline & Beeinträchtigt & $5(25,0)$ & $11(55,0)$ & 0 & $3(15,0)$ & $1(5,0)$ & \\
\hline \multirow[t]{2}{*}{ Prüfungsphase } & Normal & 0 & 0 & $2(40,0)$ & $2(40,0)$ & $1(2,0)$ & \multirow[t]{2}{*}{0,786} \\
\hline & Beeinträchtigt & 0 & $1(33,3)$ & $1(33,3)$ & 0 & $1(33,3)$ & \\
\hline \multirow{2}{*}{$\begin{array}{l}\text { Gesamtstich- } \\
\text { probe }\end{array}$} & Normal & $7(7,8)$ & $8(8,9)$ & $32(35,6)$ & $37(41,1)$ & $6(6,7)$ & \multirow[t]{2}{*}{$<0,001$} \\
\hline & Beeinträchtigt & $9(22,0)$ & $22(53,7)$ & $2(4,9)$ & $5(12,2)$ & $3(7,3)$ & \\
\hline
\end{tabular}

Tab. 7 Anzahl der Referendare mit entsprechender Musterzugehörigkeit mit normaler bzw. erhöhter Verausgabungsbereitschaft

\begin{tabular}{|c|c|c|c|c|c|c|c|}
\hline \multirow[t]{2}{*}{ Phase } & \multirow{2}{*}{$\begin{array}{l}\text { Overcom- } \\
\text { mitment }\end{array}$} & \multicolumn{5}{|c|}{ AVEM-Muster } & \multirow[t]{2}{*}{$p_{\text {Fisher's exact Test }}$} \\
\hline & & A, $n(\%)$ & $\mathrm{B}, \boldsymbol{n}(\%)$ & G, $n(\%)$ & $S, n(\%)$ & Keine Zuordnung, $n$ (\%) & \\
\hline \multirow[t]{2}{*}{ Einstiegsphase } & Normal & $3(5,1)$ & $11(18,6)$ & $16(27,1)$ & $27(45,8)$ & $2(3,4)$ & \multirow[t]{2}{*}{$<0,001$} \\
\hline & Erhöht & $4(30,8)$ & $6(46,2)$ & $2(15,4)$ & 0 & $1(7,7)$ & \\
\hline \multirow{2}{*}{$\begin{array}{l}\text { Qualifizie- } \\
\text { rungsphase }\end{array}$} & Normal & $4(11,8)$ & $4(11,8)$ & $10(29,4)$ & $12(35,3)$ & $4(11,8)$ & \multirow[t]{2}{*}{$<0,01$} \\
\hline & Erhöht & $5(29,4)$ & $8(47,1)$ & $3(17,6)$ & $1(5,9)$ & 0 & \\
\hline \multirow[t]{2}{*}{ Prüfungsphase } & Normal & 0 & 0 & $2(33,3)$ & $2(33,3)$ & $2(33,3)$ & \multirow[t]{2}{*}{0,429} \\
\hline & Erhöht & 0 & $1(50,0)$ & $1(50,0)$ & 0 & 0 & \\
\hline \multirow{2}{*}{$\begin{array}{l}\text { Gesamtstich- } \\
\text { probe }\end{array}$} & Normal & $7(7,8)$ & $15(15,2)$ & $28(28,3)$ & $41(41,4)$ & $8(8,1)$ & \multirow[t]{2}{*}{$<0,001$} \\
\hline & Erhöht & $9(22,0)$ & $15(46,9)$ & $6(18,8)$ & $1(3,1)$ & $1(3,1)$ & \\
\hline
\end{tabular}

Tab. 8 Anzahl der Referendare mit entsprechender Musterzugehörigkeit und dem Burnout-Risiko nach Kalimo

\begin{tabular}{|c|c|c|c|c|c|c|c|}
\hline \multirow[t]{2}{*}{ Phase } & \multirow{2}{*}{$\begin{array}{l}\text { Burnout-Risiko (nach } \\
\text { Kalimo klassifiziert) }\end{array}$} & \multicolumn{5}{|c|}{ AVEM-Muster } & \multirow[t]{2}{*}{$p_{\text {Fisher's exact Test }}$} \\
\hline & & A, $n(\%)$ & $\mathrm{B}, \boldsymbol{n}(\%)$ & $\mathbf{G}, \boldsymbol{n}(\%)$ & S, n (\%) & Keine Zuordnung, $\boldsymbol{n}$ (\%) & \\
\hline \multirow{3}{*}{$\begin{array}{l}\text { Einstiegs- } \\
\text { phase }\end{array}$} & Kein Risiko & $2(29)$ & $5(29)$ & $18(100)$ & $22(82)$ & $2(67)$ & \multirow[t]{3}{*}{$<0,001$} \\
\hline & Einige Symptome & $5(71)$ & $11(65)$ & 0 & $5(18)$ & $1(33)$ & \\
\hline & Burnout-Risiko & 0 & $1(6)$ & 0 & 0 & 0 & \\
\hline \multirow{3}{*}{$\begin{array}{l}\text { Qualifizie- } \\
\text { rungsphase }\end{array}$} & Kein Risiko & $7(78)$ & $4(33)$ & $13(100)$ & $8(62)$ & $2(50)$ & \multirow[t]{3}{*}{$<0,01$} \\
\hline & Einige Symptome & $2(22)$ & $7(58)$ & 0 & $5(38)$ & $2(50)$ & \\
\hline & Burnout-Risiko & 0 & $1(8)$ & 0 & 0 & 0 & \\
\hline \multirow{3}{*}{$\begin{array}{l}\text { Prüfungs- } \\
\text { phase }\end{array}$} & Kein Risiko & 0 & 0 & $2(67)$ & $2(100)$ & $1(50)$ & \multirow[t]{3}{*}{0,786} \\
\hline & Einige Symptome & 0 & $1(100)$ & $1(33)$ & 0 & $1(50)$ & \\
\hline & Burnout-Risiko & 0 & 0 & 0 & 0 & 0 & \\
\hline \multirow{3}{*}{$\begin{array}{l}\text { Gesamtstich- } \\
\text { probe }\end{array}$} & Kein Risiko & $9(56)$ & $9(30)$ & $33(97)$ & $32(76)$ & $5(56)$ & \multirow[t]{3}{*}{$<0,001$} \\
\hline & Einige Symptome & $7(44)$ & $19(63)$ & $1(3)$ & $10(24)$ & $4(44)$ & \\
\hline & Burnout-Risiko & 0 & $2(7)$ & 0 & 0 & 0 & \\
\hline
\end{tabular}

negative Korrelation zwischen geringer Distanzierungsfähigkeit bzw. erhöhter Verausgabungsbereitschaft und beeinträchtigter psychischer Gesundheit und erhöhtem Burnout-Risiko. Ebenso steigt mit sinkender offensiver Problembewältigung das Risiko beeinträchtigter psychischer Gesundheit und das BurnoutRisiko. Bei geringer sozialer Unterstützung steigt sowohl das Risiko für eine beeinträchtigte psychische Gesundheit als auch das Burnout-Risiko.

\section{Diskussion}

Diese Studie zeigt Ansatzmöglichkeiten für eine sinnvolle frühzeitige Prävention zur Gesunderhaltung von Lehramtsanwärtern auf.
Die psychische Gesundheit der Referendare ist bereits am Anfang des Berufslebens beeinträchtigt. Knapp ein Drittel klagt über eine schlechte psychische Gesundheit und eine vermehrte BurnoutSymptomatik. Das Ergebnis dieser Befragung deckt sich mit den Ergebnissen von Klusmann et al. [19], wonach die emotionale Erschöpfung als eine Kernkomponente des Burnout-Syndroms im 
Tab. 9 Partielle Korrelation zwischen den Dimensionen der Arbeitsbedingten Verhaltens- und Erlebensmustern und den Kategorien des Maslach-

Burnout-Inventars sowie der erhöhten Anstrengungsbereitschaft unter Berücksichtigung der Referendariatsphase als Kontrollvariable

\begin{tabular}{|c|c|c|c|c|c|c|c|}
\hline & & $\begin{array}{l}\text { Psychische } \\
\text { Gesundheit }\end{array}$ & $\begin{array}{l}\text { Overcommit- } \\
\text { ment }\end{array}$ & $\begin{array}{l}\text { Emotionale } \\
\text { Erschöpfung }\end{array}$ & Zynismus & $\begin{array}{l}\text { Leistungs- } \\
\text { fähigkeit }\end{array}$ & $\begin{array}{l}\text { Burnout-Risiko nach } \\
\text { Kalimo }\end{array}$ \\
\hline \multirow{2}{*}{$\begin{array}{l}\text { Subjektive Bedeutsamkeit } \\
\text { der Arbeit }\end{array}$} & $r$ & $-0,133$ & $-0,031$ & $-0,228$ & $-0,350$ & 0,182 & $-0,296$ \\
\hline & $p$ & 0,133 & 0,727 & 0,009 & $<0,001$ & 0,038 & 0,001 \\
\hline \multirow[t]{2}{*}{ Beruflicher Ehrgeiz } & $r$ & $-0,161$ & 0,028 & $-0,109$ & $-0,160$ & 0,120 & $-0,148$ \\
\hline & $p$ & 0,068 & 0,751 & 0,217 & 0,069 & 0,173 & 0,093 \\
\hline \multirow{2}{*}{$\begin{array}{l}\text { Verausgabungs- } \\
\text { bereitschaft }\end{array}$} & $r$ & 0,452 & 0,600 & 0,417 & 0,275 & $-0,171$ & 0,372 \\
\hline & $p$ & $<0,001$ & $<0,001$ & $<0,001$ & 0,002 & 0,052 & $<0,001$ \\
\hline \multirow[t]{2}{*}{ Perfektionsstreben } & $r$ & 0,072 & 0,225 & 0,024 & $-0,033$ & 0,164 & $-0,042$ \\
\hline & $p$ & 0,413 & 0,01 & 0,785 & 0,712 & 0,062 & 0,637 \\
\hline \multirow[t]{2}{*}{ Distanzierungsfähigkeit } & $r$ & $-0,58$ & $-0,776$ & $-0,518$ & $-0,340$ & 0,387 & $-0,508$ \\
\hline & $p$ & $<0,001$ & $<0,001$ & $<0,001$ & $<0,001$ & $<0,001$ & $<0,001$ \\
\hline \multirow[t]{2}{*}{ Resignationstendenz } & $r$ & 0,457 & 0,480 & 0,461 & 0,425 & $-0,457$ & 0,525 \\
\hline & $p$ & $<0,001$ & $<0,001$ & $<0,001$ & $<0,001$ & $<0,001$ & $<0,001$ \\
\hline \multirow{2}{*}{$\begin{array}{l}\text { Offensive } \\
\text { Problembewältigung }\end{array}$} & $r$ & $-0,514$ & $-0,309$ & $-0,417$ & $-0,423$ & 0,495 & $-0,510$ \\
\hline & $p$ & $<0,001$ & $<0,001$ & $<0,001$ & $<0,001$ & $<0,001$ & $<0,001$ \\
\hline \multirow{2}{*}{$\begin{array}{l}\text { Innere Ruhe und } \\
\text { Ausgeglichenheit }\end{array}$} & $r$ & $-0,391$ & $-0,419$ & $-0,343$ & $-0,347$ & 0,374 & $-0,410$ \\
\hline & $p$ & $<0,001$ & $<0,001$ & $<0,001$ & $<0,001$ & $<0,001$ & $<0,001$ \\
\hline \multirow[t]{2}{*}{ Erfolgserleben im Beruf } & $r$ & $-0,193$ & $-0,171$ & $-0,173$ & $-0,109$ & 0,152 & $-0,174$ \\
\hline & $p$ & 0,028 & 0,051 & 0,049 & 0,219 & 0,084 & 0,048 \\
\hline \multirow[t]{2}{*}{ Lebenszufriedenheit } & $r$ & $-0,588$ & $-0,400$ & $-0,477$ & $-0,551$ & 0,468 & $-0,580$ \\
\hline & $p$ & $<0,001$ & $<0,001$ & $<0,001$ & $<0,001$ & $<0,001$ & $<0,001$ \\
\hline \multirow{2}{*}{$\begin{array}{l}\text { Erleben sozialer } \\
\text { Unterstützung }\end{array}$} & $r$ & $-0,430$ & $-0,363$ & $-0,349$ & $-0,465$ & 0,244 & $-0,420$ \\
\hline & $p$ & $<0,001$ & $<0,001$ & $<0,001$ & $<0,001$ & 0,005 & $<0,001$ \\
\hline
\end{tabular}

Verlaufdes Referendariats zunimmt. Den Autoren zufolge spricht dieses Ergebnis dafür, dass der Übergang in den praktischen Teil der Lehrerausbildung eine Anpassungsleistung erfordert.

Die Verausgabungsbereitschaft ist bei einem Viertel der Referendare erhöht. Bei übermäßigem OC sind in der Literatur Zusammenhänge zur Arbeitsfähigkeit einerseits und zur psychischen Gesundheit andererseits beschrieben [25]. Eine erhöhte Anstrengungsbereitschaft ist mit einer geringeren Arbeitsfähigkeit und auch mit einem verstärkten Burnout-Risiko verbunden. In dieser Studie verfügten die Referendare mehrheitlich über die gesundheitsförderlichen Verhaltens- und Erlebensmuster G und S. Das deckt sich in etwa mit den Ergebnissen von Römer et al. [26], die bei Lehramtsstudierenden mehrheitlich das Muster S mit 35\% und das Muster G mit 29\% beobachteten. Sie schlussfolgerten, dass Lehramtsstudierende weniger engagiert sind und weniger Freude am Studium haben als Studierende anderer Fachrichtungen.

Eine ähnliche Musterverteilung bei Lehramtsstudierenden wurde auch von Rothland [27] im Zusammenhang mit der Potsdamer Lehrerstudie konstatiert. Verglichen mit der Potsdamer Stichprobe ist der Anteil risikobehafteter Verhaltensmuster unter den Lehramtsanwärtern dieser Stichprobe zwar geringer in Hinblick auf die spätere Berufstätigkeit sollten jedoch Präventionsmaßnahmen ergriffen werden, damit die Gesundheit der jetzigen Referendare und späteren Lehrkräfte erhalten bleibt. Unter den Lehrkräften der Potsdamer Lehrerstudie traten mehrheitlich die gesundheitsgefährdenden Muster A (30 \%) und B (29\%) auf. Es sollte mittels gezielter Präventionsmaßnahmen verhindert werden, dass Lehramtsstudierende bzw. Referendare im späteren Berufsleben vermehrt in gesundheitsgefährdende Muster A oder B verfallen. Laut Potsdamer Lehrerstudie geht während eines Burnout-Prozesses etwa ein Viertel des Risikomusters A in das andere Risikomuster B (Burnout) über. Etwa $23 \%$ der Lehrkräfte mit dem gesundheitsförderlichen Muster S waren zu einem späteren Untersuchungszeitpunkt in das gesundheitsgefährdende Risikomuster B gewechselt. Auch unter diesem Gesichtspunkt ist die überwiegende Musterzugehörigkeit zum Muster S in dieser Studie nicht unbedingt von Vorteil.

Alarmierend sind die Zusammenhänge der Zugehörigkeit zu den risikobehafteten Verhaltens- und Erlebensmuster mit einer beeinträchtigten psychischen Gesundheit der Referendare und sowie dem Auftreten zumindest einiger Burnout-Symptome. Klusmann et al. [19] fanden heraus, dass Referendare mit den gesundheitsförderlichen Mustern G und S geringere emotionale Erschöpfung angaben, als Referendare mit Risikomustern A und $\mathrm{B}$.

Als eine mögliche Präventionsmaßnahme bietet sich ein gezieltes Stimm- 
training an. Nusseck et al. [24] fanden heraus, dass ein Stimmtraining nicht nur die positive Wirkungen auf die Qualität und Funktionalität der Stimme hat, sondern ebenfalls bei den Referendaren positiv die Distanzierungsfähigkeit verbessert, was Schaarschmidt und Fischer [29] zufolge eine sehr wichtige protektive Komponente für die Stärkung der psychischen Gesundheit darstellt.

Da OC zu einem höheren BurnoutRisiko führen kann, ist hier ein Präventionsansatz zu erkennen, um das hohe Engagement mit gesundheitsförderlichen und primärpräventiven Maßnahmen $\mathrm{zu}$ unterstützen. Burnout wird als Folge eines bestehenden Ungleichgewichts zwischen Anforderungen und Belohnung beschrieben. Dabei spielen sowohl personale Ressourcen als auch Ressourcen aus der sozialen Umwelt (sozialemotionale, sozial-ökologische, sozialstaatliche) eine Rolle Hauptaugenmerk sollte deshalb auf der Stärkung persönlicher Ressourcen liegen. $\mathrm{Zu}$ diesen Ressourcen auf Seiten der Referendare gehören physische (z. B. stabiles Immunsystem), psychische, interaktionelle-psychische (Empathie, Beziehungsund Konfliktfähigkeit, Respekt, Verlässlichkeit, die Fähigkeit, nach Hilfe und Unterstützung zu fragen) und ökonomische Ressourcen. Besonders relevant sind bei der Entstehung von Burnout die psychischen Ressourcen. Dazu zählen spezifische Fertigkeiten und positive Persönlichkeitseigenschaften (z. B. Zuversicht, Optimismus, emotionale Stabilität, Kontrollüberzeugung, Selbstwirksamkeitserwartung). Auch eine hohe Distanzierungsfähigkeit, eine offensive Problembewältigung sowie innere $\mathrm{Ru}$ he und Ausgeglichenheit sind wichtige persönliche Ressourcen. Weitere, sehr wichtige externe Faktoren sind soziale Unterstützung sowie Erfolgserleben im Beruf. Bei einer Zugehörigkeit zu den Risikomustern $\mathrm{A}$ und $\mathrm{B}$ ist dringend eine Teilnahme an einem Kurs zum besseren Umgang mit Stress angeraten, um unterschiedliche Belastungen im Referendariat mithilfe einer breiten Palette an Bewältigungsstrategien meistern zu können [18]. Gute und ausreichende Coping-Strategien sind persönlichen Eigenschaften, die die Auswirkungen der Belastungen entsprechend des Arbeitsanforderungs-ArbeitsressourcenModell [10] bzw. der Theorie der Ressourcenerhaltung [7] abmildern.

Viele Referendare verspüren in Prüfungssituationen ein ,ängstlich-nervöses Spannungsgefühl“. Die Prüfung ist ein potenzieller Stressor im Studium und in der Referendarzeit im Sinne des transaktionalen Stressmodells von Lazarus und Folkman [21]. Die Bewertung der Prüfungssituation erfolgt vor dem Hintergrund einer persönlichen „hochgestellten Planke“, aber auch vor dem Hintergrund der familiär und gesellschaftlich geprägten Erwartungen. Diese kann man als zu erreichenden „Soll-Wert" betrachten. Die Einhaltung dieses "Sollwertes" ist entscheidend für das individuelle Wohlbefinden und den Selbstwert. Die Befürchtung einer Soll-Ist-Diskrepanz bedeutet eine stressbezogene primäre $\mathrm{Be}$ wertung der Prüfung, d.h. des „Stressereignisses“. In der Sekundärbewertung werden die personalen Ressourcen, die der Referendar besitzt oder die ihm fehlen, als Kompetenzen (z. B. Selbstwirksamkeitserwartung, gute Vorbereitung) und externe Unterstützungsmöglichkeiten (als Unterstützung durch vertraute Personen, z. B. von der Familie, von einem Kommilitonen, von einem Freund) beurteilt. Diese persönlichen Ressourcen sollten gestärkt werden (z. B. durch mehr Unterstützung durch den Mentor), um Prüfungsängste abzumildern.

Als ein einfacher Schritt im Zuge der Prävention könnte gleich zu Beginn des Studiums der AVEM-Fragebogen ausgefüllt werden. Als Ergebnis kann dann zielgerecht geschult werden, wie mit einem gesundheitsförderlichen Verhalten der Risikoausprägung rechtzeitig gegengesteuert werden kann. Dazu müssen Möglichkeiten einer Intervention an den Hochschulen geschaffen werden.

Weitere Präventionsmöglichkeiten zur Senkung der Beanspruchung sind zum einen eine bessere Vorbereitung auf das Referendariat durch die Vermittlung eines fundierten Wissens über Klassenführung oder auch eine längere praktische Erfahrung (Geben von Nachhilfe, Leiten von Jugendgruppen) während des Studiums [19]. Zum anderen unterstreichen Drüge et al. [13] die
Wichtigkeit von sozialer Unterstützung, sozialen Beziehungen und eine positive Feedbackkultur für die Gesunderhaltung der Lehramtsanwärter im Vorbereitungsdienst, was bei der Arbeit in den Schulen umgesetzt werden muss.

\section{Limitierungen}

Die Befragung beschränkt sich auf den Raum Magdeburg und lässt sich nicht ohne weiteres auf andere Bundesländer übertragen. Darüber hinaus wurden Belastungsfaktoren nicht explizit abgefragt, sodass ein Zusammenhang zwischen konkreter Belastung und emotionaler Erschöpfung als Folge nicht ermittelt werden kann.

Diese Studie bildet außerdem nur einen Querschnitt an einer kleinen Stichprobe ab. Wünschenswert sind Längsschnittuntersuchungen, um tatsächlich vorhandene Unterschiede zwischen einzelnen Phasen des Referendariats im intraindividuellen Vergleich feststellen zu können.

\section{Fazit für die Praxis}

\section{- Bereits im Studium müssen gesund- heitsförderliche Verhaltensweisen vermittelt bzw. sinnvolle präventive Maßnahmen wie Stressbewälti- gungskurse oder autogenes Training angeboten werden. \\ - Während des Referendariats soll- ten Präventionsmaßnahmen in den einmal wöchentlich stattfindenden Seminaren (speziell im Hauptsemi- nar) etabliert werden. \\ - Kurse zum Erlernen von offensiver Problembewältigung können sinn- voll sein, um die Entwicklung eines Burnout-Syndroms zu verhindern. \\ - Die soziale Unterstützung und eine Feedbackkultur im Referendariat müssen gewährleistet sein, um die emotionale Erschöpfung der Referendare zu vermeiden.}




\section{Korrespondenzadresse}

\section{Dr. Sabine Darius}

Bereich Arbeitsmedizin, Medizinische Fakultät, Otto-von-Guericke-Universität Magdeburg Leipziger Str. 44, 39120 Magdeburg,

Deutschland

sabine.darius@med.ovgu.de

Funding. Open Access funding provided by Projekt DEAL.

\section{Einhaltung ethischer Richtlinien}

Interessenkonflikt. S. Darius, K. Bunzel, E. EhmsCiechanowicz und I. Böckelmann geben an, dass kein Interessenkonflikt besteht.

Für diesen Beitrag wurden von den Autoren keine Studien an Menschen oder Tieren durchgeführt. Für die aufgeführten Studien gelten die jeweils dort angegebenen ethischen Richtlinien. Die Befragung wurde mit Zustimmung der Ethikkommission der Medizinischen Fakultät der Otto-von-Guericke-Universität (Registrierungsnummer 19/16) durchgeführt.

Open Access Dieser Artikel wird unter der Creative Commons Namensnennung 4.0 International Lizenz veröffentlicht, welche die Nutzung, Vervielfältigung, Bearbeitung, Verbreitung und Wiedergabe in jeglichem Medium und Format erlaubt, sofern Sie den/die ursprünglichen Autor(en) und die Quelle ordnungsgemäß nennen, einen Link zur Creative Commons Lizenz beifügen und angeben, ob Änderungen vorgenommen wurden.

Die in diesem Artikel enthaltenen Bilder und sonstiges Drittmaterial unterliegen ebenfalls der genannten Creative Commons Lizenz, sofern sich aus der Abbildungslegende nichts anderes ergibt. Sofern das betreffende Material nicht unter der genannten Creative Commons Lizenz steht und die betreffende Handlung nicht nach gesetzlichen Vorschriften erlaubt ist, ist für die oben aufgeführten Weiterverwendungen des Materials die Einwilligung des jeweiligen Rechteinhabers einzuholen.

Weitere Details zur Lizenz entnehmen Sie bitte der Lizenzinformation auf http://creativecommons.org/ licenses/by/4.0/deed.de.

\section{Literatur}

1. Bailer J, Schwarz D, Witthöft M, Stübinger C, Rist F (2008) Prävalenz psychischer Syndrome bei Studierenden einer deutschen Universität. Psychother Psychosom Med Psychol 58:423-429

2. Bauer J, Unterbrink T, Hack A, Pfeifer R, BuhlGriesshaber V, Müller U, Wesche H, Frommhold M Seibt R, Scheuch K, Wirsching M (2007) Working conditions, adverse events and mental health problems in a sample of 949 German teachers. Int Arch Occup Environ Health 80(5):442-449. https:// doi.org/10.1007/s00420-007-0170-7

3. Bergmann C, Muth T, Loerbroks A (2019) Medical students' perceptions of stress due to academic studies and its interrelationships with other domains of life. A qualitative study. Med Educ
Online 24(1):1603526. https://doi.org/10.1080/ 10872981.2019.1603526

4. Bonde JPE (2008) Psychosocial factors at work and risk of depression. A systematic review of the epidemiological evidence. Occup Environ Med65(7):438-445. https://doi.org/10.1136/oem. 2007.038430

5. Borrelli I, Benevene P, Fiorilli C, D'Amelio F, Pozzi G (2014) Working conditions and mental health in teachers: a preliminary study. Occup Med 64(7):530-532. https://doi.org/10.1093/occmed/ kqu108

6. Brandl-Bredenbeck HP, Kämpfe A, Köster C (2013) Gesundheit von Lehramtsstudierenden - Ausgewählte Ergebnisse einer empirischen Untersuchung an der Universität Paderborn. In: Marchwacka MA (Hrsg) Gesundheitsförderung im Setting Schule. Springer VS, Wiesbaden, S 329-345

7. Buchwald P, Hobfoll SE (2013) Die Theorie der Ressourcenerhaltung: Implikationen für den Zusammenhang von Stress und Kultur. In: Genkova P (Hrsg) Handbuch Stress und Kultur. Interkulturelle und kulturvergleichende Perspektiven. Springer VS, Wiesbaden, S127-138

8. Christ O, van Dick R, Wagner U (2004) Belastung und Beanspruchung beiLehrern in der Ausbildung. In: Hillert A, Schmitz E (Hrsg) Psychosomatische Erkrankungen bei Lehrerinnen und Lehrern. [Ursachen, Folgen, Lösungen]. Schattauer, Stuttgart S113-119

9. Ciechanowicz EK (2015) Betriebliches Eingliederungsmanagement bei Lehrkräften in SachsenAnhalt (Dissertation, Otto-von-Guericke-Universität)

10. Demerouti E, Nachreiner F (2019) Zum Arbeitsanforderungen-Arbeitsressourcen-Modell von Burnout und Arbeitsengagement - Stand der Forschung. Z Arb Wiss 73(2):119-130. https://doi org/10.1007/s41449-018-0100-4

11. DESTATIS (2018) Zahl der Pensionierungen von Lehrkräften 2017 erneut rückläufig. https://www. destatis.de/DE/Presse/Pressemitteilungen/2018/ 12/PD18_509_742.html.Zugegriffen:20.07.2020

12. Dragano N, Siegrist J, Nyberg ST et al (2017) Effortreward imbalance at work and incident coronary heart disease. A multicohort study of 90,164 individuals. Epidemiology 28(4):619-626. https:// doi.org/10.1097/EDE.0000000000000666

13. Drüge M, Schleider K, Rosati A-S (2015) Psychosoziale Belastungen im Referendariat - Merkmale, Ausprägungen, Folgen. Dtsch Sch 106:358-372

14. Gluschkoff K, Elovainio M, Kinnunen U, Mullola S, Hintsanen M, Keltikangas-Jarvinen L, Hintsa T (2016) Work stress, poor recovery and burnout in teachers. Occup Med (Lond) 66(7):564-570. https://doi.org/10.1093/occmed/kqw086

15. Goldberg DP, Gater R, Sartorius N, Ustun TB, Piccinelli M, Gureje O, Rutter C (1997) The validity of two versions of the GHQ in the WHO study of mental illness in general health care. Psychol Med 27:191-197

16. Hinz A, Zenger M, Brähler E, Spitzer S, Scheuch $K_{\text {, }}$ Seibt R (2014) Effort-reward imbalance and mental health problems in 1074 German teachers, compared with those in the general population. Stress Health. https://doi.org/10.1002/smi.2596

17. Kalimo R, Pahkin K, Mutanen P, Toppinen-Tanner S (2003) Staying well or burning out at work: Work characteristics and personal resources as long-term predictors. Work Stress 17(2):109-122. https://doi.org/10.1080/0267837031000149919

18. Karing C, Beelmann A (2016) Implementation und Evaluation eines multimodalen Stressbewältigungstrainings bei Lehramtsstudierenden.
Z Gesundheitspsychol 24(2):89-101. https://doi. org/10.1026/0943-8149/a000159

19. Klusmann U, Kunter M, Voss T, Baumert J (2012) Berufliche Beanspruchung angehender Lehrkräfte: Die Effekte von Persönlichkeit, pädagogischer Vorerfahrung und professioneller Kompetenz. ZPädagog Psychol 26(4):275-290. https://doi.org/ 10.1024/1010-0652/a000078

20. Kristiansen J, Lund SP, Persson R, Shibuya $H$, Nielsen PM, Scholz M (2014) A study of classroom acoustics and school teachers' noise exposure, voice load and speaking time during teaching, and the effects on vocal and mental fatigue development. Int Arch Occup Environ Health 87(8):851-860. https://doi.org/10.1007/s00420 014-0927-8

21. Lazarus RS, Folkmann S (1984) Stress, appraisal, and coping. Springer, New York

22. Linden M, Maier W, Achberger M, Herr R, Helmchen H, Benkert O (1996) Psychische Erkrankungen und ihre Behandlng in Allgemeinarztpraxen in Deutschland. Ergebnisse aus einer Studie der Weltgesundheitsorganisation (WHO). Nervenarzt 67:205-215

23. Maslach C, Jackson SE, Leiter MP (Hrsg) (1996) MBI manual, 3. Aufl. Consulting Psychologists Press, Palo Alto

24. Nusseck M, Richter B, Echternach M, Spahn C (2017) Psychologische Effekte eines präventiven Stimmtrainings im Lehramtsreferendariat. HNO 65(7):599-609. https://doi.org/10.1007/s00106016-0157-3

25. Preckel D, von Känel R, Kudielka BM, Fischer JE (2005) Overcommitment to work is associated with vital exhaustion. Int Arch Occup Environ Health 78(2):117-122. https://doi.org/10.1007/s00420004-0572-8

26. Römer J, Appel J, Drews F, Rauin U (2012) Burnout-Risiko von Lehramts- und Jurastudierenden der Anfangssemester. Praev Gesundheitsf 7(3):203-208. https://doi.org/10.1007/s11553012-0345-2

27. Rothland M (2011) Risikomerkmale von Lehramtsstudierenden. Z f Bildungsforsch 1(3):179-197. https://doi.org/10.1007/s35834-011-0016-y

28. Schaarschmidt U (2005) Halbtagsjobber? Psychische Gesundheit im Lehrerberuf - Analyse eines veränderungsbedürftigen Zustandes. Beltz, Weinheim

29. Schaarschmidt U, Fischer A (2008) Arbeitsbezogenes Verhaltens- und Erlebensmuster. Handanweisung, 3. Aufl. Dr. G. Schuhfried, Frankfurt/M

30. Scheuch K, Haufe E, Seibt R (2015) Lehrergesundheit. Dtsch Arztebl 112(20):347-356

31. Schöne K, Schäfer M, Dreyer P, Sommer-SchickertK, Rose D-M (2016) Akustische Situation in Schulen - Erfahrungen und Erkenntnisse aus dem Institut für Lehrergesundheit. Arbeitsmed Sozialmed Umweltmed 51:724-729

32. Seibt R, Spitzer S, Blank M, Scheuch K (2009) Predictors of work ability in occupations with psychological stress. J Public Health 17(1):9-18 https://doi.org/10.1007/s10389-008-0194-9

33. Seibt R, Hübler A, Steputat A, Scheuch K (2012) Verausgabungs-Belohnungs-Verhältnis und Burnout-Risiko bei Lehrerinnen und Ärztinnen - ein Berufsgruppenvergleich. Arbeitsmed Sozialmed Umweltmed 47:396-406

34. Shimizu M, Wada K, Wang G, Kawashima M, Yoshino $Y$, Sakaguchi $H$, Ohta H, Miyaoka H, Aizawa $Y$ (2011) Factors of working conditions and prolonged fatigue among teachers at public elementary and junior high schools. Ind Health 49:434-442 


\section{Originalarbeit}

35. Siegrist J (1996) Adverse health effects of higheffort/low-reward conditions. J Occup Health Psychol 1(1):27-41

36. Vedovato TG, Monteiro I (2014) Health conditions and factors related to the work ability of teachers. Ind Health 52:121-128 